\title{
Synthesis of New [8Fe-7S] Clusters: A Topological Link Between the Core Structures of P-Cluster, FeMo-co, and FeFe-co of Nitoregenases
}

\author{
Yasuhiro Ohki, Yohei Ikagawa, and Kazuyuki Tatsumi \\ Department of Chemistry, Graduate School of Science and Research Center for \\ Materials Science, Nagoya University, Furo-cho, Chikusa-ku, Nagoya 464-8602, Japan
}

Supporting Information

Contents:

1. Cyclic voltammetry of cluster 2

2. EPR spectrum of cluster $\mathbf{3}$ observed with contaminated cluster 2

3. Molecular structures and selected bond distances $(\AA)$ and angles $\left(^{\circ}\right)$

3-1. complex 1

3-2. cluster 2

3-3. cluster 3 


\section{Cyclic voltammetry of cluster 2}

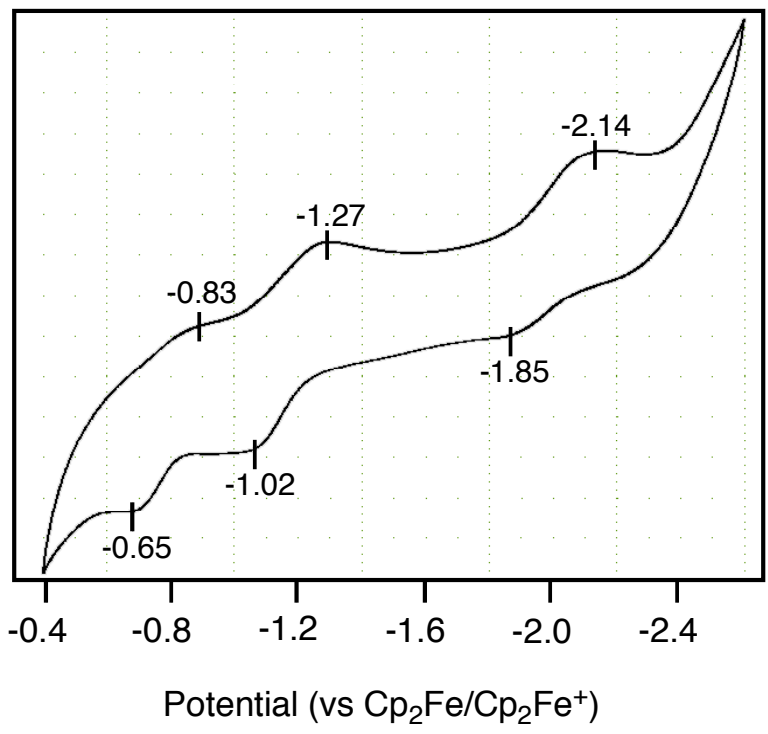

Condition: $2 \mathrm{mM}$ of 2 in THF, scan $0.1 \mathrm{~V} / \mathrm{s}$, ambient temperature,

supporting electrolyte; $0.2 \mathrm{M}\left(\mathrm{NBu}_{4}\right)\left(\mathrm{PF}_{6}\right) \quad$ working electrode; glassy carbon counter electrode; platinum

\section{2. $\quad$ EPR spectrum of cluster 3 observed with contaminated cluster 2}

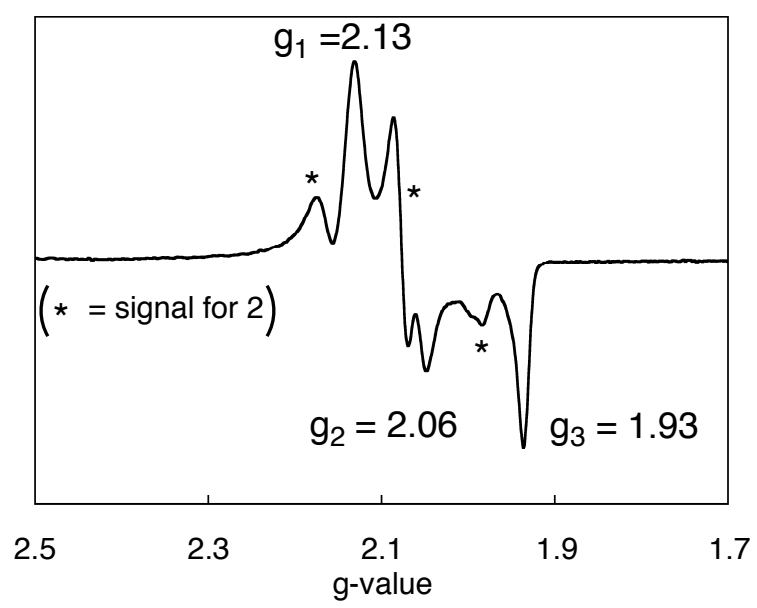

Condition: X-band, $0.8 \mathrm{mM}$ in frozen toluene, $15 \mathrm{~K}$, microwave power; $1.0 \mathrm{~mW} \quad$ modulation; $\mathrm{Fq}=100 \mathrm{kHz}$, Width $=1.0 \mathrm{mT}$ 
3-1. Molecular structure and selected bond distances $(\AA)$ and angles $\left(^{\circ}\right)$ for complex 1

Molecular structure of $\mathbf{1}$ with thermal ellipsoids at $50 \%$ probability level.

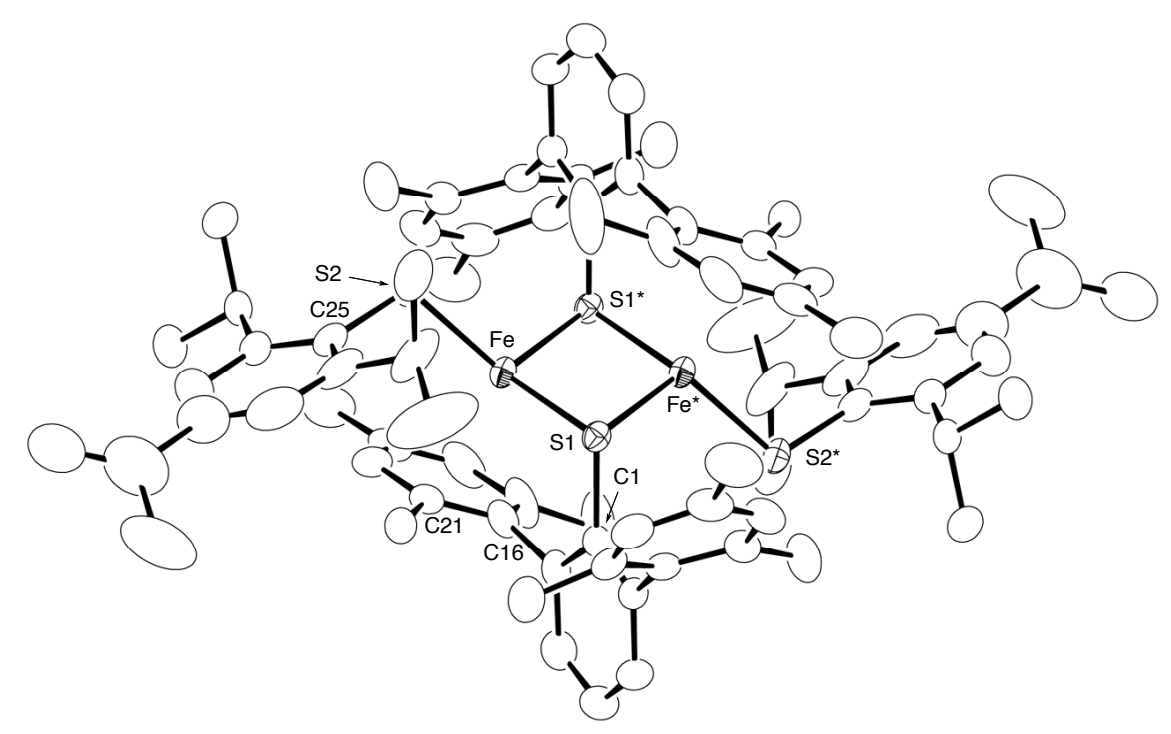

Table S-1. Selected bond distances $(\AA)$ and angles $\left(^{\circ}\right)$ for $\mathbf{1}$.

\begin{tabular}{|c|c|c|c|}
\hline $\mathrm{Fe}-\mathrm{Fe}^{*}$ & $3.0099(5)$ & $\mathrm{S} 1-\mathrm{Fe}-\mathrm{S} 1^{*}$ & $101.52(2)$ \\
\hline Fe-S1 & $2.3694(7)$ & S1-Fe-S2 & $122.16(3)$ \\
\hline $\mathrm{Fe}-\mathrm{S} 1^{*}$ & $2.3888(7)$ & $\mathrm{S} 1^{*}-\mathrm{Fe}-\mathrm{S} 2$ & $111.92(2)$ \\
\hline $\mathrm{Fe}-\mathrm{S} 2$ & $2.2734(8)$ & $\mathrm{Fe}-\mathrm{S} 1-\mathrm{Fe}^{*}$ & $78.48(2)$ \\
\hline $\mathrm{Fe}-\mathrm{C} 16$ & $2.650(2)$ & $\mathrm{Fe}-\mathrm{S} 1-\mathrm{C} 1$ & $107.95(9)$ \\
\hline \multirow[t]{2}{*}{$\mathrm{Fe}-\mathrm{C} 21$} & $2.532(2)$ & $F e^{*}-S 1-C 1$ & $107.29(8)$ \\
\hline & & $\mathrm{Fe}-\mathrm{S} 2-\mathrm{C} 25$ & $112.90(9)$ \\
\hline
\end{tabular}


3-2. Molecular structure and selected bond distances ( $(̊)$ and angles $\left(^{\circ}\right)$ for cluster 2

Molecular structure of $\mathbf{2}$ with thermal ellipsoids at $50 \%$ probability level.

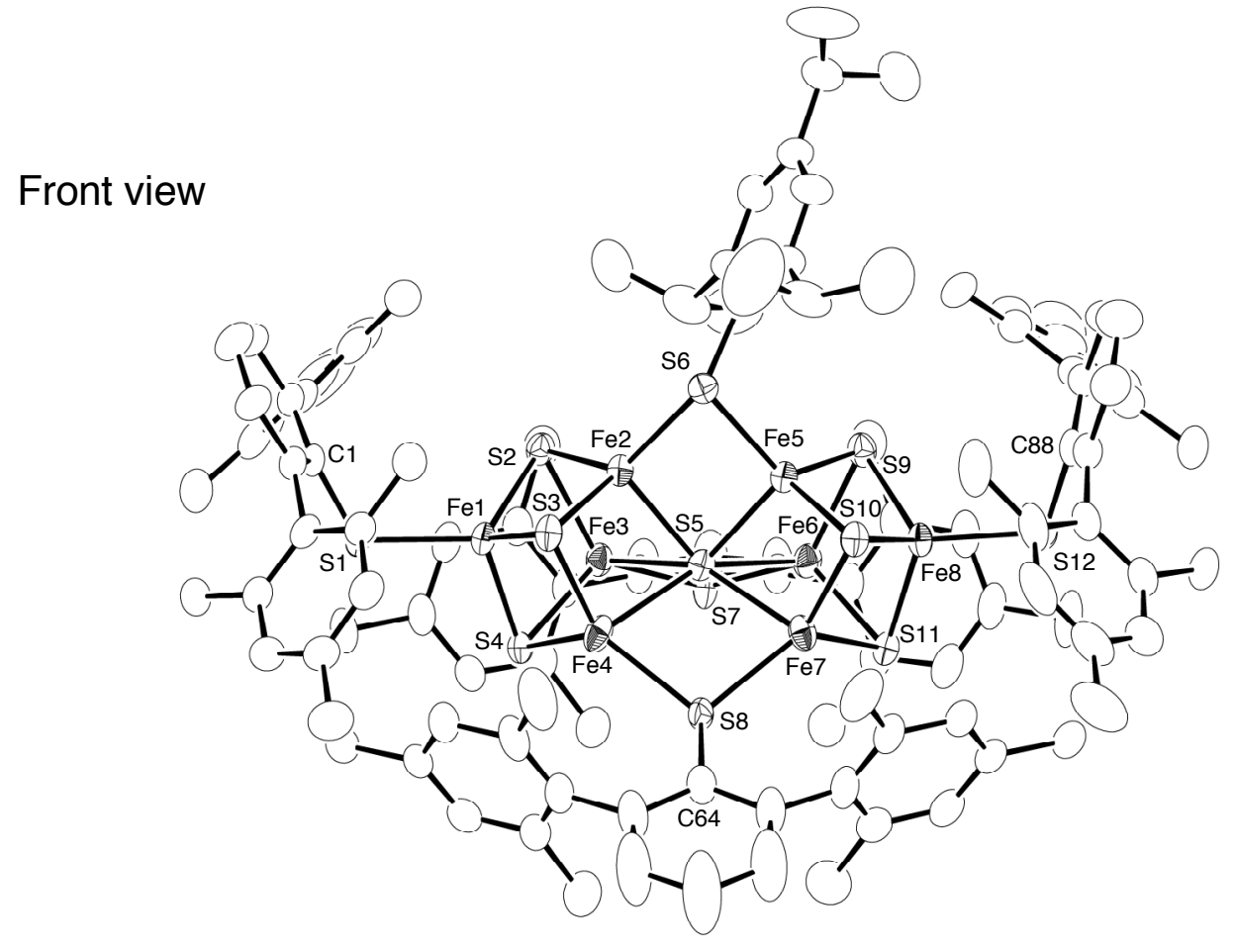

Top view

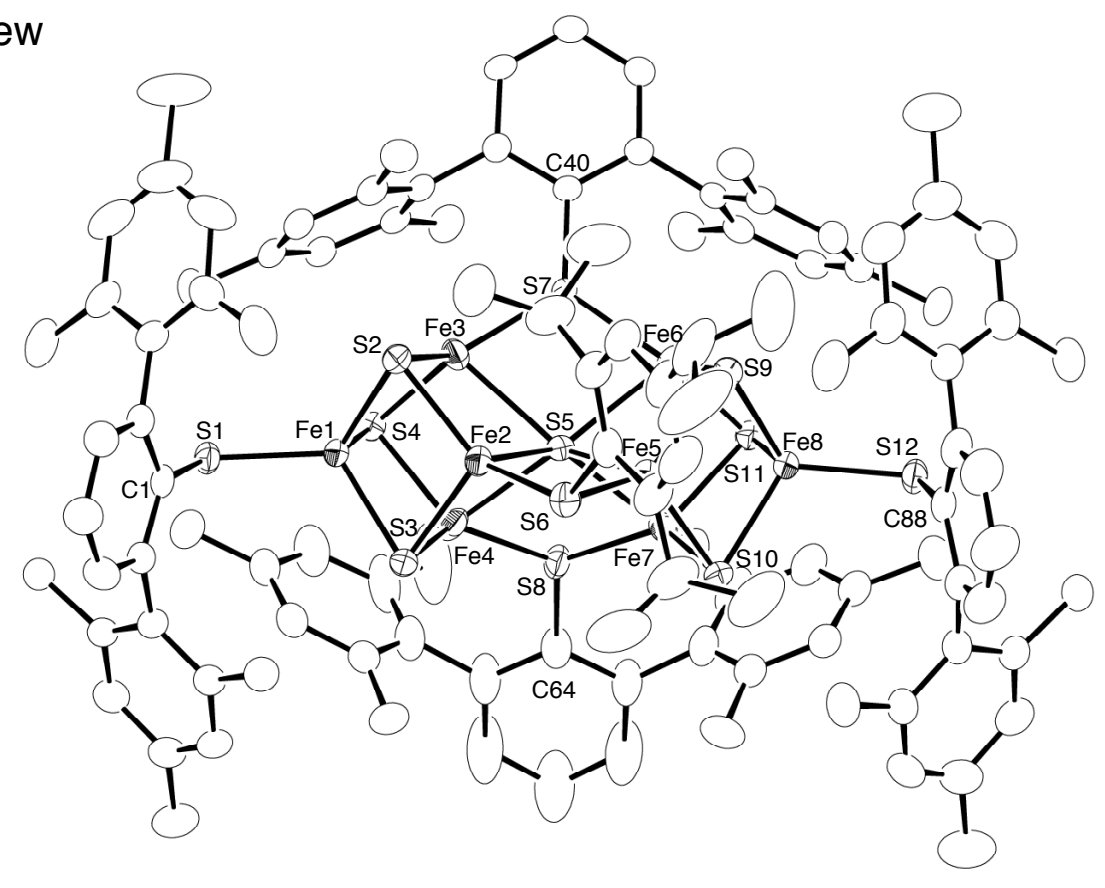


Table S-2. Selected bond distances $(\AA)$ and angles $\left({ }^{\circ}\right)$ for 2 .

\begin{tabular}{|c|c|c|c|c|c|c|c|c|c|c|}
\hline $\mathrm{Fe}(1)$ & $\mathrm{Fe}(2)$ & $2.7456(9)$ & $S(1)$ & $\mathrm{Fe}(1)$ & $S(2)$ & 118.94(5) & $\mathrm{Fe}(1)$ & $S(1)$ & $C(1)$ & $116.06(13)$ \\
\hline $\mathrm{Fe}(1)$ & $\mathrm{Fe}(3)$ & $2.7919(9)$ & $S(1)$ & $\mathrm{Fe}(1)$ & $S(3)$ & $117.64(4)$ & & & & \\
\hline $\mathrm{Fe}(1)$ & $\mathrm{Fe}(4)$ & $2.7782(11)$ & $S(1)$ & $\mathrm{Fe}(1)$ & $S(4)$ & $107.37(4)$ & $\mathrm{Fe}(1)$ & $\mathrm{S}(2)$ & $\mathrm{Fe}(2)$ & $74.48(4)$ \\
\hline $\mathrm{Fe}(2)$ & $\mathrm{Fe}(3)$ & $2.7871(8)$ & $\mathrm{S}(2)$ & $\mathrm{Fe}(1)$ & $S(3)$ & $103.33(4)$ & $\mathrm{Fe}(1)$ & $S(2)$ & $\mathrm{Fe}(3)$ & $75.31(4)$ \\
\hline $\mathrm{Fe}(2)$ & $\mathrm{Fe}(4)$ & $2.9521(9)$ & $S(2)$ & $\mathrm{Fe}(1)$ & $S(4)$ & $103.37(4)$ & $\mathrm{Fe}(2)$ & $S(2)$ & $\mathrm{Fe}(3)$ & $75.58(4)$ \\
\hline $\mathrm{Fe}(2)$ & $\mathrm{Fe}(5)$ & $2.9103(10)$ & $S(3)$ & $\mathrm{Fe}(1)$ & $S(4)$ & $104.45(4)$ & & & & \\
\hline $\mathrm{Fe}(3)$ & $\mathrm{Fe}(4)$ & $2.9873(10)$ & & & & & $\mathrm{Fe}(1)$ & $\mathrm{S}(3)$ & $\mathrm{Fe}(2)$ & $73.89(4)$ \\
\hline $\mathrm{Fe}(3)$ & $\mathrm{Fe}(6)$ & $3.7050(10)$ & $\mathrm{S}(2)$ & $\mathrm{Fe}(2)$ & $S(3)$ & $104.27(4)$ & $\mathrm{Fe}(1)$ & $\mathrm{S}(3)$ & $\mathrm{Fe}(4)$ & $74.80(3)$ \\
\hline $\mathrm{Fe}(4)$ & $\mathrm{Fe}(7)$ & $3.6196(10)$ & $\mathrm{S}(2)$ & $\mathrm{Fe}(2)$ & $S(5)$ & $107.68(4)$ & $\mathrm{Fe}(2)$ & $\mathrm{S}(3)$ & $\mathrm{Fe}(4)$ & $80.54(4)$ \\
\hline $\mathrm{Fe}(5)$ & $\mathrm{Fe}(6)$ & $2.8102(8)$ & $\mathrm{S}(2)$ & $\mathrm{Fe}(2)$ & $S(6)$ & $129.07(4)$ & & & & \\
\hline $\mathrm{Fe}(5)$ & $\mathrm{Fe}(7)$ & $2.7906(8)$ & $S(3)$ & $\mathrm{Fe}(2)$ & $S(5)$ & $100.49(4)$ & $\mathrm{Fe}(1)$ & $S(4)$ & $\mathrm{Fe}(3)$ & $75.62(3)$ \\
\hline $\mathrm{Fe}(5)$ & $\mathrm{Fe}(8)$ & $2.6947(10)$ & $S(3)$ & $\mathrm{Fe}(2)$ & $S(6)$ & $110.78(4)$ & $\mathrm{Fe}(1)$ & $S(4)$ & $\mathrm{Fe}(4)$ & $75.50(3)$ \\
\hline $\mathrm{Fe}(6)$ & $\mathrm{Fe}(7)$ & $2.9140(10)$ & $S(5)$ & $\mathrm{Fe}(2)$ & $S(6)$ & $101.03(4)$ & $\mathrm{Fe}(3)$ & $S(4)$ & $\mathrm{Fe}(4)$ & $81.72(4)$ \\
\hline $\mathrm{Fe}(6)$ & $\mathrm{Fe}(8)$ & $2.7871(10)$ & & & & & & & & \\
\hline \multirow[t]{3}{*}{$\mathrm{Fe}(7)$} & $\mathrm{Fe}(8)$ & $2.7584(9)$ & $\mathrm{S}(2)$ & $\mathrm{Fe}(3)$ & $S(4)$ & $102.13(4)$ & $\mathrm{Fe}(2)$ & $S(5)$ & $\mathrm{Fe}(3)$ & $72.10(4)$ \\
\hline & & & $\mathrm{S}(2)$ & $\mathrm{Fe}(3)$ & $S(5)$ & $102.34(4)$ & $\mathrm{Fe}(2)$ & $S(5)$ & $\mathrm{Fe}(4)$ & $76.92(4)$ \\
\hline & & & $\mathrm{S}(2)$ & $\mathrm{Fe}(3)$ & $S(7)$ & $133.22(4)$ & $\mathrm{Fe}(2)$ & $S(5)$ & $\mathrm{Fe}(5)$ & $78.59(3)$ \\
\hline $\mathrm{Fe}(1)$ & $S(1)$ & $2.2336(13)$ & $S(4)$ & $\mathrm{Fe}(3)$ & $S(5)$ & $99.76(4)$ & $\mathrm{Fe}(2)$ & $S(5)$ & $\mathrm{Fe}(6)$ & $129.95(5)$ \\
\hline $\mathrm{Fe}(1)$ & $S(2)$ & $2.2795(12)$ & $S(4)$ & $\mathrm{Fe}(3)$ & $S(7)$ & $124.50(4)$ & $\mathrm{Fe}(2)$ & $S(5)$ & $\mathrm{Fe}(7)$ & $133.90(4)$ \\
\hline $\mathrm{Fe}(1)$ & $S(3)$ & $2.2876(12)$ & $S(5)$ & $\mathrm{Fe}(3)$ & $S(7)$ & $75.39(4)$ & $\mathrm{Fe}(3)$ & $S(5)$ & $\mathrm{Fe}(4)$ & $75.58(3)$ \\
\hline \multirow[t]{2}{*}{$\mathrm{Fe}(1)$} & $S(4)$ & $2.2628(11)$ & & & & & $\mathrm{Fe}(3)$ & $S(5)$ & $\mathrm{Fe}(5)$ & $133.82(5)$ \\
\hline & & & $\mathrm{S}(3)$ & $\mathrm{Fe}(4)$ & $S(4)$ & $104.09(5)$ & $\mathrm{Fe}(3)$ & $\mathrm{S}(5)$ & $\mathrm{Fe}(6)$ & $99.58(4)$ \\
\hline $\mathrm{Fe}(2)$ & $S(2)$ & $2.2576(13)$ & $S(3)$ & $\mathrm{Fe}(4)$ & $S(5)$ & $96.18(4)$ & $\mathrm{Fe}(3)$ & $S(5)$ & $\mathrm{Fe}(7)$ & $150.95(4)$ \\
\hline $\mathrm{Fe}(2)$ & $\mathrm{S}(3)$ & $2.2802(12)$ & $S(3)$ & $\mathrm{Fe}(4)$ & $S(8)$ & $135.47(4)$ & $\mathrm{Fe}(4)$ & $S(5)$ & $\mathrm{Fe}(5)$ & $131.08(4)$ \\
\hline $\mathrm{Fe}(2)$ & $S(5)$ & $2.3002(12)$ & $S(4)$ & $\mathrm{Fe}(4)$ & $S(5)$ & $99.90(4)$ & $\mathrm{Fe}(4)$ & $S(5)$ & $\mathrm{Fe}(6)$ & $150.59(4)$ \\
\hline \multirow[t]{2}{*}{$\mathrm{Fe}(2)$} & $S(6)$ & $2.3296(11)$ & $\mathrm{S}(4)$ & $\mathrm{Fe}(4)$ & $S(8)$ & $120.44(4)$ & $\mathrm{Fe}(4)$ & $S(5)$ & $\mathrm{Fe}(7)$ & $96.04(4)$ \\
\hline & & & $S(5)$ & $\mathrm{Fe}(4)$ & $S(8)$ & $77.14(4)$ & $\mathrm{Fe}(5)$ & $S(5)$ & $\mathrm{Fe}(6)$ & $73.13(3)$ \\
\hline $\mathrm{Fe}(3)$ & $S(2)$ & $2.2903(12)$ & & & & & $\mathrm{Fe}(5)$ & $S(5)$ & $\mathrm{Fe}(7)$ & $72.42(3)$ \\
\hline $\mathrm{Fe}(3)$ & $S(4)$ & $2.2912(12)$ & $S(5)$ & $\mathrm{Fe}(5)$ & $S(6)$ & $102.03(4)$ & $\mathrm{Fe}(6)$ & $S(5)$ & $\mathrm{Fe}(7)$ & $73.96(4)$ \\
\hline $\mathrm{Fe}(3)$ & $S(5)$ & $2.4322(13)$ & $S(5)$ & $\mathrm{Fe}(5)$ & $S(9)$ & $105.13(4)$ & & & & \\
\hline \multirow[t]{2}{*}{$\mathrm{Fe}(3)$} & $S(7)$ & $2.3235(12)$ & $\mathrm{S}(5)$ & $\mathrm{Fe}(5)$ & $S(10)$ & $105.99(4)$ & $\mathrm{Fe}(2)$ & $S(6)$ & $\mathrm{Fe}(5)$ & $77.87(3)$ \\
\hline & & & $S(6)$ & $\mathrm{Fe}(5)$ & $\mathrm{S}(9)$ & $120.70(4)$ & $\mathrm{Fe}(2)$ & $S(6)$ & $C(25)$ & $131.52(16)$ \\
\hline $\mathrm{Fe}(4)$ & $S(3)$ & $2.2867(11)$ & $S(6)$ & $\mathrm{Fe}(5)$ & $S(10)$ & $115.53(4)$ & $\mathrm{Fe}(5)$ & $S(6)$ & $C(25)$ & $103.69(14)$ \\
\hline $\mathrm{Fe}(4)$ & $S(4)$ & $2.2750(12)$ & $S(9)$ & $\mathrm{Fe}(5)$ & $S(10)$ & $105.96(5)$ & & & & \\
\hline $\mathrm{Fe}(4)$ & $S(5)$ & $2.4429(12)$ & & & & & $\mathrm{Fe}(3)$ & $S(7)$ & $\mathrm{Fe}(6)$ & $105.73(4)$ \\
\hline \multirow[t]{2}{*}{$\mathrm{Fe}(4)$} & $S(8)$ & $2.3299(14)$ & $S(5)$ & $\mathrm{Fe}(6)$ & $S(7)$ & $75.64(4)$ & $\mathrm{Fe}(3)$ & $S(7)$ & $\mathrm{C}(40)$ & $122.77(16)$ \\
\hline & & & $S(5)$ & $\mathrm{Fe}(6)$ & $S(9)$ & $100.22(4)$ & $\mathrm{Fe}(6)$ & $S(7)$ & $\mathrm{C}(40)$ & $122.92(14)$ \\
\hline $\mathrm{Fe}(5)$ & $\mathrm{S}(5)$ & $2.2953(11)$ & $S(5)$ & $\mathrm{Fe}(6)$ & $S(11)$ & $101.64(4)$ & & & & \\
\hline $\mathrm{Fe}(5)$ & $S(6)$ & $2.3015(12)$ & $S(7)$ & $\mathrm{Fe}(6)$ & $S(9)$ & $131.90(5)$ & $\mathrm{Fe}(4)$ & $S(8)$ & $\mathrm{Fe}(7)$ & $102.10(4)$ \\
\hline $\mathrm{Fe}(5)$ & $S(9)$ & $2.2479(13)$ & $S(7)$ & $\mathrm{Fe}(6)$ & $S(11)$ & $125.17(4)$ & $\mathrm{Fe}(4)$ & $S(8)$ & $C(64)$ & $121.15(17)$ \\
\hline $\mathrm{Fe}(5)$ & $S(10)$ & $2.2512(13)$ & $\mathrm{S}(9)$ & $\mathrm{Fe}(6)$ & $S(11)$ & $102.78(4)$ & $\mathrm{Fe}(7)$ & $\mathrm{S}(8)$ & $C(64)$ & $121.27(16)$ \\
\hline $\mathrm{Fe}(6)$ & $S(5)$ & $2.4191(12)$ & $S(5)$ & $\mathrm{Fe}(7)$ & $S(8)$ & $77.60(4)$ & $\mathrm{Fe}(5)$ & $S(9)$ & $\mathrm{Fe}(6)$ & $76.70(4)$ \\
\hline $\mathrm{Fe}(6)$ & $S(7)$ & $2.3241(12)$ & $S(5)$ & $\mathrm{Fe}(7)$ & $S(10)$ & $100.79(4)$ & $\mathrm{Fe}(5)$ & $S(9)$ & $\mathrm{Fe}(8)$ & $72.99(4)$ \\
\hline $\mathrm{Fe}(6)$ & $\mathrm{S}(9)$ & $2.2813(11)$ & $S(5)$ & $\mathrm{Fe}(7)$ & $S(11)$ & $101.84(4)$ & $\mathrm{Fe}(6)$ & $\mathrm{S}(9)$ & $\mathrm{Fe}(8)$ & $75.27(4)$ \\
\hline \multirow[t]{2}{*}{$\mathrm{Fe}(6)$} & $S(11)$ & $2.2863(14)$ & $S(8)$ & $\mathrm{Fe}(7)$ & $S(10)$ & $135.36(4)$ & & & & \\
\hline & & & $S(8)$ & $\mathrm{Fe}(7)$ & $S(11)$ & $120.51(4)$ & $\mathrm{Fe}(5)$ & $S(10)$ & $\mathrm{Fe}(7)$ & $75.91(3)$ \\
\hline $\mathrm{Fe}(7)$ & $S(5)$ & $2.4253(14)$ & $S(10)$ & $\mathrm{Fe}(7)$ & $S(11)$ & $103.64(4)$ & $\mathrm{Fe}(5)$ & $S(10)$ & $\mathrm{Fe}(8)$ & $72.97(4)$ \\
\hline $\mathrm{Fe}(7)$ & $\mathrm{S}(8)$ & $2.3237(12)$ & & & & & $\mathrm{Fe}(7)$ & $S(10)$ & $\mathrm{Fe}(8)$ & $74.33(3)$ \\
\hline $\mathrm{Fe}(7)$ & $S(10)$ & $2.2859(10)$ & $S(9)$ & $\mathrm{Fe}(8)$ & $S(10)$ & $103.86(4)$ & & & & \\
\hline \multirow[t]{2}{*}{$\mathrm{Fe}(7)$} & $S(11)$ & $2.2730(12)$ & $S(9)$ & $\mathrm{Fe}(8)$ & $S(11)$ & $103.57(4)$ & $\mathrm{Fe}(6)$ & $S(11)$ & $\mathrm{Fe}(7)$ & $79.46(4)$ \\
\hline & & & $S(9)$ & $\mathrm{Fe}(8)$ & $S(12)$ & $117.39(4)$ & $\mathrm{Fe}(6)$ & $S(11)$ & $\mathrm{Fe}(8)$ & $75.62(4)$ \\
\hline $\mathrm{Fe}(8)$ & $\mathrm{S}(9)$ & $2.2828(14)$ & $S(10)$ & $\mathrm{Fe}(8)$ & $S(11)$ & $104.24(4)$ & $\mathrm{Fe}(7)$ & $S(11)$ & $\mathrm{Fe}(8)$ & $74.97(3)$ \\
\hline $\mathrm{Fe}(8)$ & $S(10)$ & $2.2803(12)$ & $S(10)$ & $\mathrm{Fe}(8)$ & $S(12)$ & $115.13(4)$ & & & & \\
\hline $\mathrm{Fe}(8)$ & $S(11)$ & $2.2599(10)$ & $S(11)$ & $\mathrm{Fe}(8)$ & $S(12)$ & $111.22(4)$ & $\mathrm{Fe}(8)$ & $S(12)$ & $C(88)$ & $110.97(15)$ \\
\hline $\mathrm{Fe}(8)$ & $S(12)$ & 2.2272(13) & & & & & & & & \\
\hline
\end{tabular}


3-3. Molecular structure and selected bond distances $(\AA)$ and angles $\left(^{\circ}\right)$ for cluster 3

Molecular structure of $\mathbf{3}$ with thermal ellipsoids at $50 \%$ probability level.
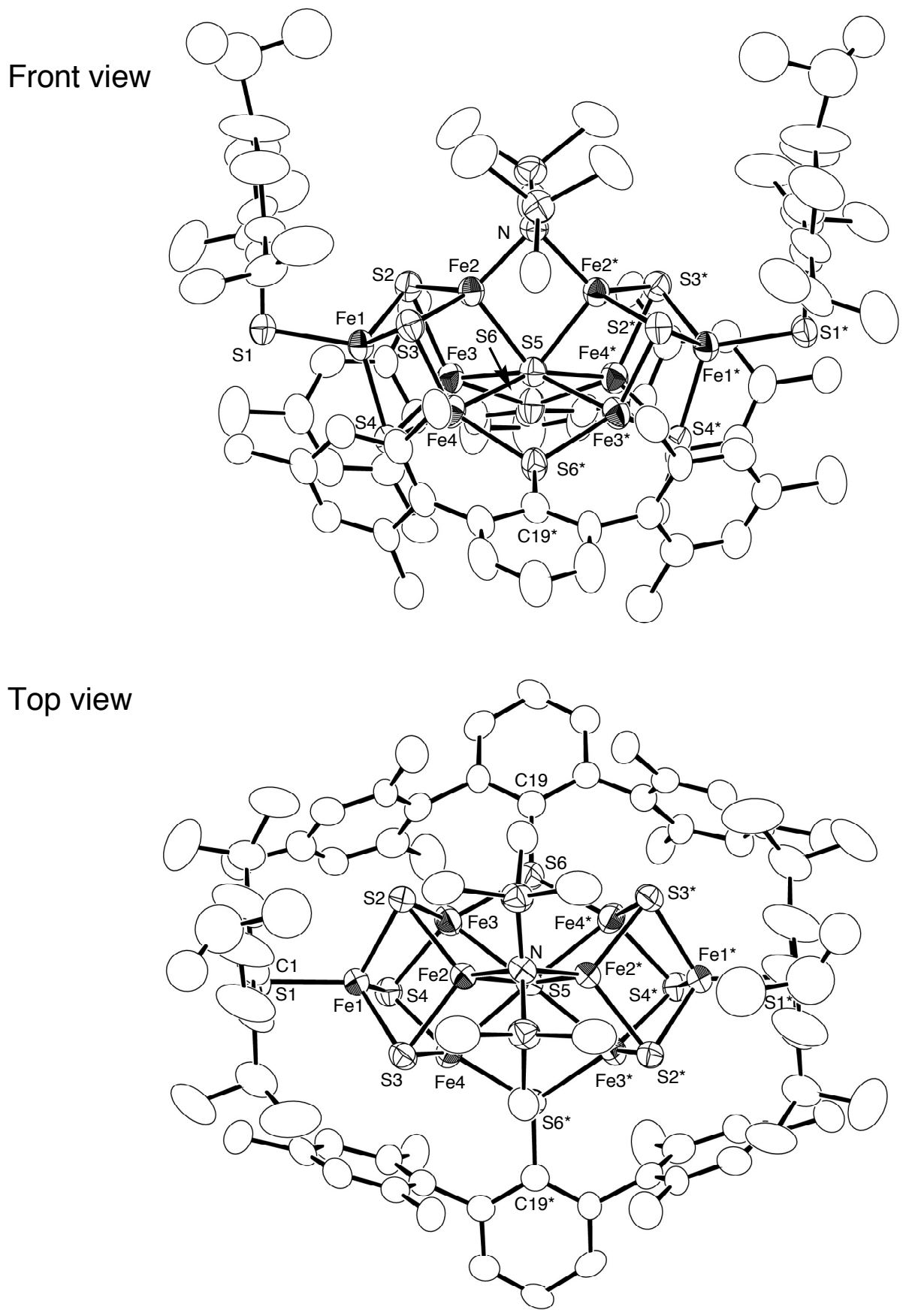
Table S-3. Selected bond distances $(\AA)$ and angles $\left({ }^{\circ}\right)$ for 3.

\begin{tabular}{|c|c|c|c|c|c|c|c|}
\hline Fe1-Fe2 & $2.763(2)$ & Fe1-S1 & $2.232(2)$ & S1-Fe1-S2 & $113.83(14)$ & $\mathrm{Fe} 2-\mathrm{N}-\mathrm{Fe}^{*}$ & $86.7(4)$ \\
\hline $\mathrm{Fe} 1-\mathrm{Fe} 3$ & $2.7575(18)$ & Fe1-S2 & $2.269(3)$ & S1-Fe1-S3 & $113.89(12)$ & & \\
\hline Fe1-Fe4 & $2.771(2)$ & Fe1-S3 & $2.266(4)$ & S1-Fe1-S4 & $115.28(10)$ & Fe1-S1-C1 & $100.2(3)$ \\
\hline Fe2-Fe3 & $2.860(2)$ & Fe1-S4 & $2.265(2)$ & S2-Fe1-S3 & $102.87(11)$ & & \\
\hline $\mathrm{Fe} 2-\mathrm{Fe} 4$ & $2.854(2)$ & & & S2-Fe1-S4 & $105.04(10)$ & Fe1-S2-Fe2 & $74.75(12)$ \\
\hline \multirow[t]{2}{*}{ Fe3-Fe4 } & $2.983(3)$ & Fe2-S2 & $2.282(4)$ & S3-Fe1-S4 & $104.66(14)$ & Fe1-S2-Fe3 & $74.57(10)$ \\
\hline & & Fe2-S3 & $2.267(2)$ & & & Fe2-S2-Fe3 & 77.61(12) \\
\hline $\mathrm{Fe} 2-\mathrm{Fe} 2^{*}$ & $2.804(2)$ & Fe2-S5 & $2.324(3)$ & S2-Fe2-S3 & 102.44(12) & & \\
\hline \multirow[t]{21}{*}{$\mathrm{Fe} 3-\mathrm{Fe} 4^{*}$} & $3.677(2)$ & $\mathrm{Fe} 2-\mathrm{N}$ & $2.042(8)$ & S2-Fe2-S5 & $104.08(9)$ & Fe1-S3-Fe2 & $75.12(12)$ \\
\hline & & & & S2-Fe2-N & $122.06(13)$ & Fe1-S3-Fe4 & $75.07(11)$ \\
\hline & & Fe3-S2 & $2.283(3)$ & S3-Fe2-S5 & $104.61(9)$ & Fe2-S3-Fe4 & $77.71(9)$ \\
\hline & & Fe3-S4 & 2.292(2) & S3-Fe2-N & $121.43(14)$ & & \\
\hline & & Fe3-S5 & $2.428(2)$ & S5-Fe2-N & $99.6(2)$ & Fe1-S4-Fe3 & $74.46(9)$ \\
\hline & & Fe3-S6 & $2.317(2)$ & & & Fe1-S4-Fe4 & $74.82(10)$ \\
\hline & & & & S2-Fe3-S4 & $103.74(9)$ & Fe3-S4-Fe4 & $81.11(10)$ \\
\hline & & Fe4-S3 & $2.283(2)$ & S2-Fe3-S5 & $100.82(13)$ & & \\
\hline & & Fe4-S4 & $2.296(4)$ & S2-Fe3-S6 & $128.03(11)$ & Fe2-S5-Fe2* & $74.20(11)$ \\
\hline & & Fe4-S5 & $2.4296(15)$ & S4-Fe3-S5 & $99.54(12)$ & Fe2-S5-Fe3 & $73.97(6)$ \\
\hline & & Fe4-S6* & $2.320(3)$ & S4-Fe3-S6 & $128.06(11)$ & Fe2-S5-Fe3* & $129.99(11)$ \\
\hline & & & & S5-Fe3-S6 & $76.90(10)$ & Fe2-S5-Fe4 & $73.76(5)$ \\
\hline & & & & & & Fe2-S5-Fe4* & $128.88(11)$ \\
\hline & & & & S3-Fe4-S4 & $103.14(13)$ & Fe3-S5-Fe3* & $153.43(16)$ \\
\hline & & & & S3-Fe4-S5 & $100.82(11)$ & Fe3-S5-Fe4 & $75.76(7)$ \\
\hline & & & & S3-Fe4-S6* & $130.02(15)$ & Fe3-S5-Fe4* & $98.38(7)$ \\
\hline & & & & S4-Fe4-S5 & $99.42(10)$ & $\mathrm{Fe} 4-\mathrm{S} 5-\mathrm{Fe} 4 *$ & $154.80(16)$ \\
\hline & & & & S4-Fe4-S6* & $126.69(11)$ & & \\
\hline & & & & S5-Fe4-S6* & $76.81(8)$ & Fe3-S6-Fe4* & 104.92(14) \\
\hline & & & & & & Fe3-S6-C19 & $124.4(3)$ \\
\hline & & & & & & Fe4-S6-C19 & 124.3(3) \\
\hline
\end{tabular}

